\title{
NY-ESO-1 is a sensitive and specific immunohistochemical marker for myxoid and round cell liposarcomas among related mesenchymal myxoid neoplasms
}

\author{
Jessica A Hemminger and O Hans Iwenofu \\ Department of Pathology and Laboratory Medicine, The Ohio State University Wexner Medical Center, \\ Columbus, OH, USA
}

\begin{abstract}
Myxoid and round cell liposarcomas constitute approximately one-third of all liposarcomas, a relatively common group of fat-derived soft tissue sarcomas. The histomorphology is a continuum between highly differentiated myxoid and poorly differentiated round cell components. The gold standard of diagnosis is dependent on histomorphology and/or identification of $t(12 ; 16)(q 13 ; p 11)$ translocation by cytogenetics or demonstration of DDIT3 rearrangements by fluorescence in situ hybridization. There are currently no diagnostic immunohistochemical stains available. The broad range of myxoid neoplasms in the differential diagnosis includes a variety of sarcomas. Given the notable differences in disease biology among myxoid neoplasms, which range from benign to aggressive, an accurate diagnosis is imperative for proper treatment and prognostication. Prompted by our recent study showing frequent expression of the cancer testis antigen NYESO-1 in myxoid and round cell liposarcomas, we sought to evaluate the utility of NY-ESO-1 as an immunohistochemical marker for myxoid and round cell liposarcoma among mesenchymal myxoid neoplasms within the differential diagnosis. Formalin-fixed, paraffin-embedded blocks were obtained for the following mesenchymal myxoid neoplasms $(n=138)$ : myxoid and round cell liposarcoma $(n=38)$; well-differentiated liposarcoma $(n=12)$; lipoma $(n=20 ; 4$ with myxoid change); extra-cardiac soft tissue myxoma $(n=39)$; extraskeletal myxoid chondrosarcoma $(n=12)$; myxofibrosarcoma $(n=10: 5$ low grade, 2 intermediate grade, 3 high grade); and low-grade fibromyxoid sarcoma $(n=7)$. Utilizing standard immunohistochemistry protocols, full sections were stained with NY-ESO-1 (clone E978), and staining was assessed for intensity (1-2+), percentage of tumor positivity, and location. In all, $36 / 38(95 \%)$ of the myxoid and round cell liposarcomas demonstrated NY-ESO-1 immunoreactivity. The majority of the positive cases $(34 / 36 ; 94 \%)$ showed strong, homogenous staining ( $>50 \%$ tumor positivity), and two cases $(6 \%)$ showed weak $(1+$ intensity), patchy staining (20-30\% tumor positivity). Immunoreactivity was predominantly cytoplasmic. All the other neoplasms evaluated were negative for NY-ESO-1. NY-ESO-1 appears to be a sensitive and a specific marker for myxoid and round cell liposarcoma among mesenchymal myxoid neoplasms. The assessment of NY-ESO-1 expression by immunohistochemistry in the appropriate setting provides a cheaper, faster, and more accessible confirmatory test.
\end{abstract}

Modern Pathology (2013) 26, 1204-1210; doi:10.1038/modpathol.2013.65; published online 19 April 2013

Keywords: differential diagnosis; immunohistochemistry; mesenchymal myxoid neoplasms; myxoid and round cell liposarcomas; NY-ESO-1

Myxoid and round cell liposarcomas constitute approximately one-third of all liposarcomas, a

Correspondence: Dr OH Iwenofu, Department of Pathology and Laboratory Medicine, The Ohio State University Wexner Medical Center, 410 West 10th Avenue, Columbus, OH 43210, USA.

Email: Hans.Iwenofu@osumc.edu

Received 4 December 2012; revised 15 January 2013; accepted 16 January 2013; published online 19 April 2013 relatively common group of fat-derived soft tissue sarcomas. ${ }^{1}$ The peak incidence of myxoid and round cell liposarcoma is in the fifth decade, and it preferentially involves the lower extremities. ${ }^{2}$ The histomorphology is described as a continuum between a highly differentiated myxoid component and a poorly differentiated round cell component. The myxoid component consists of bland, round to fusiform cells as well as a variable number of 
lipoblasts in a background of myxoid matrix and a characteristic delicate capillary network. The round cell component is composed of hypercellular sheets of primitive round cells with a high nuclear/cytoplasmic ratio and the loss of myxoid background. Increased proportion of the round cell component $(\geq 5 \%)$ is associated with adverse clinical outcomes. ${ }^{3}$ Currently, the diagnosis of myxoid and round cell liposarcoma is mostly based on histomorphology, as well as the identification of the reciprocal translocation between the DDIT3 gene on chromosome 12 and the FUS gene on chromosome $16, t(12 ; 16)(q 13 ; p 11)$ or, less commonly, a translocation between the DDIT3 and EWS genes, t(12;22)(q13;p11). ${ }^{4}$ Myxoid and round cell liposarcoma, similar to the other liposarcoma subtypes, is treated by surgical excision with or without chemoradiation.

The broad category of myxoid neoplasms includes a variety of soft tissue tumors, many of which can show a spectrum of myxoid morphology and phenotypic mimicry with myxoid and round cell liposarcoma. Given the notable differences in disease biology among myxoid neoplasms, which range from benign to aggressive, an accurate diagnosis is imperative for proper treatment and prognostication. Relevant neoplasms within the differential diagnosis of myxoid and round cell liposarcomas include other mesenchymal neoplasms that show myxoid morphology, such as extra-cardiac soft tissue myxomas, myxofibrosarcomas, low-grade fibromyxoid sarcomas, extraskeletal myxoid chondrosarcomas, well-differentiated liposarcomas, and lipomas, particularly those with prominent myxoid alteration. Currently, there is no reliable sensitive and specific immunohistochemical marker that distinguishes myxoid and round cell liposarcoma from these other soft tissue myxoid neoplasms.

Cancer testis antigens are a unique family of antigens that have largely restricted expression to testicular germ cells in a normal adult in addition to aberrant expression in a variety of neoplasms. ${ }^{5,6}$ Cancer testis antigen expression has important therapeutic implications given their innate immunogenicity, which elicits spontaneous T-cell and/or humoral responses that target the cancer testis antigen-expressing tumor cells. ${ }^{5}$ NY-ESO-1 is a highly immunogenic cancer testis antigen that is present in a variety of malignancies, including melanoma, sarcomas, and carcinomas. ${ }^{7-10}$ Prompted by recent studies showing frequent expression of the cancer testis antigen NY-ESO-1 in myxoid and round cell liposarcoma, we sought to evaluate the utility of NY-ESO-1 as an immunohistochemical marker for this tumor type among mesenchymal myxoid neoplasms within the differential diagnosis, including extra-cardiac soft tissue myxomas, myxofibrosarcomas, low-grade fibromyxoid sarcomas, well-differentiated liposarcomas, lipomas, and extraskeletal myxoid chondrosarcoma. ${ }^{11,12}$

\section{Materials and methods}

\section{Case Material}

A variety of myxoid neoplasms from 1992 to 2012 were retrospectively identified from the surgical pathology archives at the Wexner Medical Center at The Ohio State University. The cases were rereviewed by a bone and soft tissue pathologist (OHI), and the diagnoses were confirmed by histomorphology per established morphological criteria ${ }^{2}$ as well as immunohistochemistry and molecular studies where appropriate. Of the 38 cases of myxoid and round cell liposarcoma used in the study, 13 cases had been confirmed by positive DDIT3 gene rearrangement determined by fluorescence in situ hybridization and/or karyotype analysis demonstrating a $\mathrm{t}(12 ; 16)(\mathrm{q} 13 ; \mathrm{p} 11)$ translocation. A representative formalin-fixed, paraffinembedded block was obtained for each tumor (total $n=138$ ) and submitted for NY-ESO-1 immunohistochemical staining. The tumor types included are myxoid and round cell liposarcoma $(n=38)$; extra-cardiac myxoma $(n=39)$; lipoma $(n=20$; 4 with focal myxoid change); extraskeletal myxoid chondrosarcoma $\quad(n=12)$; $\quad$ well-differentiated liposarcoma $(n=12)$; myxofibrosarcoma $(n=10$ : 5 low grade, 2 intermediate grade, 3 high grade); and low-grade fibromyxoid sarcoma $(n=7)$. Of note, 18 cases of myxoid and round cell liposarcoma and 10 cases of well-differentiated liposarcoma were included from a previous study reporting NY-ESO-1 immunoreactivity. ${ }^{12}$

\section{Immunohistochemistry}

NY-ESO-1, clone E978 (Santa Cruz Biotechnology; Santa Cruz, CA, USA), was titered on normal testis, and testis was used as control tissue. Paraffinembedded tissue was cut at 4 microns, placed in a $60{ }^{\circ} \mathrm{C}$ oven for $1 \mathrm{~h}$, cooled, and then deparaffinized and rehydrated through xylenes and graded ethanol solutions to water. All slides were quenched for $5 \mathrm{~min}$ in a $3 \%$ hydrogen peroxide solution in water to block for endogenous peroxidase. Antigen retrieval was performed by heat-induced epitope retrieval, in which the slides were placed in a $1 \times$ solution of Target Retrieval Solution (Dako, Carpinteria, CA, USA) for $25 \mathrm{~min}$ at $96^{\circ} \mathrm{C}$ using a vegetable steamer and cooled for $15 \mathrm{~min}$ in solution. Slides were then placed on a Dako Autostainer Immunostaining System. The primary antibody NY-ESO-1 was diluted with a Dako antibody diluent at 1:100 and incubated for $60 \mathrm{~min}$. Slides were then blocked for endogenous biotin using the Dako Biotin Blocking System. The secondary antibody, a 1:200 dilution of biotinylated goat anti-mouse (Vector, Burlingame, CA, USA) prepared with Vector 2\% Normal Goat Serum in Dako antibody diluent, was incubated for $30 \mathrm{~min}$ at room temperature. The detection system used was 
Vectastain Elite (Vector) for 30 min. Staining was visualized with the DAB + chromogen (Dako; 5-min development). Slides were then counterstained in Richard Allen hematoxylin and dehydrated through graded ethanol solutions.

\section{Quantitation of Immunohistochemistry}

NY-ESO-1 immunopositivity was scored semiquantitatively for the percentage of tumor cells staining, intensity ( 0 , negative; $1+$, weak to moderate; $2+$, strong), and subcellular location (cytoplasmic and/ or nuclear).

\section{Results}

The results of NY-ESO-1 immunohistochemical staining are summarized in Tables 1 and 2. Consistent immunoreactivity was noted in myxoid and round cell liposarcomas (36/38; 95\% of total cases). The vast majority of the positive cases (34/36; $94 \%$ of positive cases) showed strong, homogenous staining, defined by $>50 \%$ tumor positivity. More so, $86 \%(31 / 36)$ of positive cases demonstrated $\geq 75 \%$ tumor positivity. Two cases $(6 \%$ of positive cases) showed weak, patchy immunoreactivity characterized as $1+$ staining intensity of $20 \%$ and $30 \%$ of the tumor cells. Two cases (5\% of total cases) were negative for NY-ESO-1, and the diagnosis was confirmed in both the cases by the detection of DDIT3 gene rearrangements by fluorescence in situ

Table 1 Results of NY-ESO-1 immunohistochemical staining

\begin{tabular}{lc}
\hline Neoplasm & $\begin{array}{c}\text { Positive cases } \\
\text { (percentage of total) }\end{array}$ \\
\hline Myxoid and round cell liposarcoma & $36 / 38^{\mathrm{a}}(95 \%)$ \\
Well-differentiated liposarcoma & $0 / 12^{\mathrm{a}(0 \%)}$ \\
Extra-cardiac myxoma & $0 / 39(0 \%)$ \\
Lipoma & $0 / 20(0 \%)$ \\
Extraskeletal myxoid chondrosarcoma & $0 / 12(0 \%)$ \\
Myxofibrosarcoma & $0 / 10(0 \%)$ \\
Low-grade fibromyxoid sarcoma & $0 / 7(0 \%)$
\end{tabular}

${ }^{\mathrm{a}} 18$ myxoid and round cell and 10 well-differentiated liposarcomas were previously reported. ${ }^{12}$

Table 2 Positive NY-ESO-1 staining in myxoid and round cell liposarcomas

\begin{tabular}{lcc}
\hline $\begin{array}{l}\text { Percentage of tumor } \\
\text { positivity }\end{array}$ & $\begin{array}{c}\text { Staining } \\
\text { intensity }\end{array}$ & $\begin{array}{c}\text { Number (percentage of } \\
\text { total positive) }\end{array}$ \\
\hline$\geq 75 \%$ & $2+$ & $31 / 36(86 \%)$ \\
50 to $<75 \%$ & $2+$ & $3 / 36(8 \%)$ \\
25 to $<50 \%$ & $1+$ & $1 / 36(3 \%)$ \\
5 to $<25 \%$ & $1+$ & $1 / 36(3 \%)$ \\
\hline
\end{tabular}

hybridization. The staining distribution in the myxoid and round cell liposarcomas was predominantly nuclear with less cytoplasmic staining (Figure 1). There was no difference in staining intensity or distribution between the myxoid and round cell components. No cases of extra-cardiac myxoma (0/39), lipoma (0/20), well-differentiated liposarcoma $(n=12)$, extraskeletal myxoid chondrosarcoma (0/12), myxofibrosarcoma (0/10), or low-grade fibromyxoid sarcoma (0/7) demonstrated NY-ESO-1 immunopositivity (Figure 2).

\section{Discussion}

NY-ESO-1, encoded by the CTAG1B gene on Xq28, is a highly immunogenic cancer testis antigen., ${ }^{8,13}$ NY-ESO-1 expression in normal tissue is restricted to testicular germ cells with immunoreactivity predominantly localized to the cytoplasm with occasional nuclear staining. ${ }^{10}$ In general, cancer testis antigens are thought to be involved in germ cell self-renewal or differentiation, conferring qualities such as immortality, self renewal, migratory ability, and capacity for neoplastic transformation upon expression. ${ }^{8,14,15}$ Recent reports have shown that NY-ESO-1 expression in hepatocellular carcinoma is associated with disease recurrence following surgical resection, possibly mediated by enhanced migratory ability conferred by NY-ESO- $1 .{ }^{16}$ Furthermore, the same study and others have implicated NY-ESO-1 as a possible prognostic marker in certain tumor types. ${ }^{8,16,17}$

Importantly, the expression of cancer testis antigens by tumors enables the use of targeted immunotherapy, which has shown promise in clinical studies. ${ }^{18-21}$ Variable NY-ESO-1 expression has been described in many malignancies, including melanoma, neuroblastoma, sarcomas, and a variety of carcinomas, such as esophageal, breast, prostate, lung, endometrial, ovarian, uterine, and bladder. ${ }^{8,9,22}$ Regarding mesenchymal neoplasms, previous studies have described NY-ESO-1 expression in liposarcomas, synovial sarcomas, gastrointestinal stromal tumors, uterine leiomyosarcomas, angiosarcomas, and malignant fibrous histiocytomas. ${ }^{6,23,24}$ Recently, a comprehensive study of NY-ESO-1 expression in mesenchymal tumors showed consistent, homogenous staining in synovial sarcomas $(82 \%)$ and only infrequent $(<10 \%)$ immunoreactivity in malignant peripheral nerve sheath tumors, dermatofibrosarcoma protuberans, angiosarcomas, gastrointestinal stromal tumors, chondrosarcoma, dedifferentiated liposarcoma, and undifferentiated sarcomas. ${ }^{25}$ They reported no immunoreactivity in leiomyosarcomas, solitary fibrous tumors, cellular schwannomas, Ewing sarcomas, malignant mesotheliomas, osteosarcomas, an alveolar soft part sarcoma, a highgrade spindle cell sarcoma, or a rhabdomyosarcoma. 

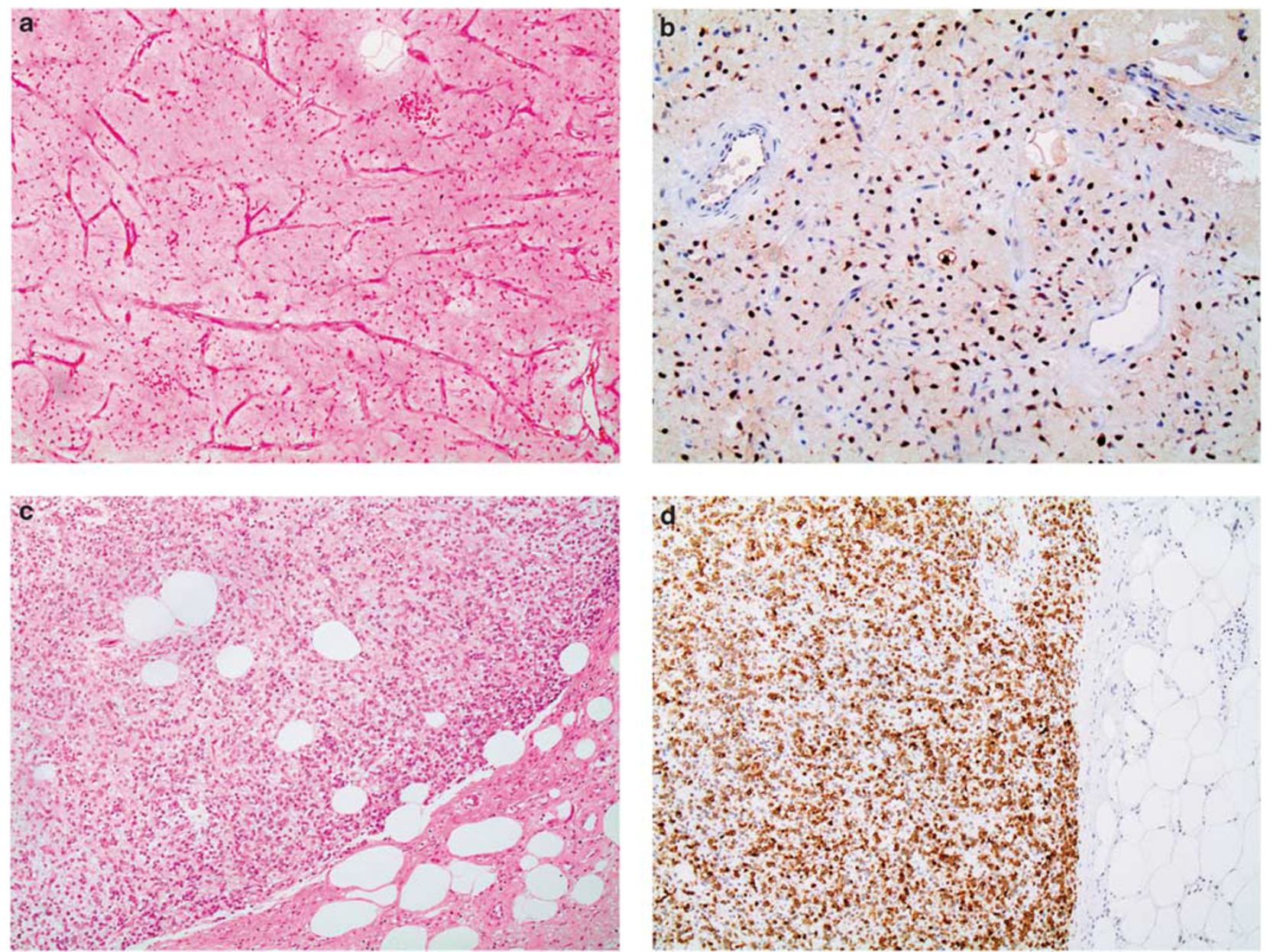

Figure 1 NY-ESO-1 immunoreactivity in myxoid and round cell liposarcoma. Myxoid component ((a); H\&E $\times 100)$ with diffuse, homogenous NY-ESO-1 immunoreactivity $((\mathbf{b}) ; \times 200)$. Round cell component $((\mathbf{c}) ; \mathrm{H} \& \mathrm{E} \times 100)$ with diffuse, homogenous NY-ESO-1 immunoreactivity $((\mathbf{d}) ; \times 100)$.

In our previous study, we reported that $7 / 8$ $(88 \%)$ and $16 / 18(89 \%)$ myxoid and round cell liposarcomas expressed CTAG1B and NY-ESO-1 by quantitative real-time PCR and immunohistochemistry, respectively. ${ }^{12}$ We also noted variable positive staining in a subset of pleomorphic liposarcomas (3/6) and an instance of strong, diffuse staining in dedifferentiated liposarcoma (1/10). Pollack et al. ${ }^{11}$ also reported NY-ESO-1 expression in 25/25 (100\%) myxoid and round cell liposarcomas evaluated by immunohistochemistry and quantitative real-time PCR. Both reports describe NY-ESO-1 expression as predominantly homogenous, which is notable given that cancer testis antigens usually demonstrate a heterogeneous staining pattern within a particular tumor. ${ }^{5,11,12,26}$ In addition to possibly enabling an enhanced response to cancer testis antigen-directed immunotherapy, diffuse staining by NY-ESO-1 increases its appeal as an immunohistochemical marker of myxoid and round cell liposarcoma.

In our current study, we expanded our cohort of myxoid and round cell liposarcomas and report NY-ESO-1 positivity in 36 of 38 (95\%) cases, characterized as strong and homogenous in $94 \%$ of the positive tumors. There was no staining in extra-cardiac soft tissue myxomas, lipomas, welldifferentiated liposarcomas, extraskeletal myxoid chondrosarcomas, myxofibrosarcomas, or low-grade fibromyxoid sarcomas. To the best of our knowledge, this is the first evaluation of differential NY-ESO-1 expression in mesenchymal myxoid neoplasms vis-à-vis myxoid and round cell liposarcomas. Our findings support the use of NY-ESO-1 as a diagnostic marker within the differential diagnosis of mesenchymal myxoid neoplasms. Currently, the diagnosis of myxoid and round cell liposarcoma is dependent on classic histomorphology and, in select cases, confirmatory molecular and/or cytogenetic studies evaluating for DDIT3 gene rearrangements and $\mathrm{t}(12 ; 16)(\mathrm{q} 13 ; \mathrm{p} 11)$, respectively. On occasion, problematic cases may arise due to a limited biopsy in which the diagnostic features are not apparent or when exclusion of other neoplasms in the differential diagnosis is difficult. The assessment of NY-ESO-1 expression by immunohistochemistry provides a less expensive, more expedient, and 

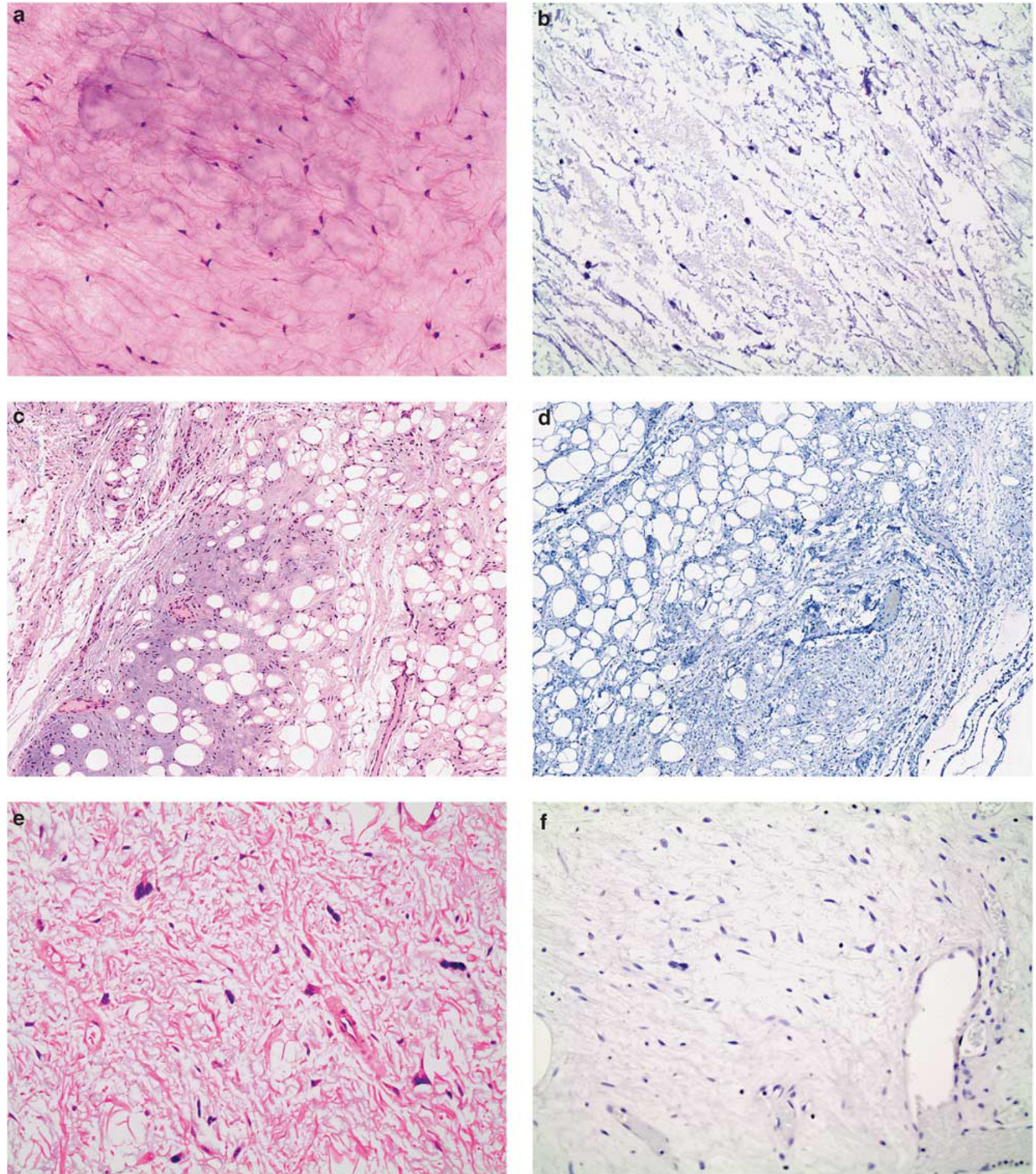

Figure 2 Negative immunoreactivity in extra-cardiac myxoma ((a) H\&E; (b) NY-ESO-1; $\times 200)$, lipoma with focal myxoid change ((c) H\&E; (d) NY-ESO-1; × 100), well-differentiated liposarcoma ((e) H\&E; (f) NY-ESO-1; $\times 200)$, extraskeletal myxoid chondrosarcoma ((g) H\&E; (h) NY-ESO-1; × 200), myxofibrosarcoma ((i) H\&E; (j) NY-ESO-1; × 200), and low-grade fibromyxoid sarcoma ((k) H\&E; (l) NY-ESO$1 ; \times 100)$.

more accessible confirmatory test, of particular utility in low-resource settings.

In conclusion, we have further validated the high frequency of strong, homogenous NY-ESO-1 expres- sion in myxoid and round cell liposarcomas in addition to demonstrating the diagnostic utility of NY-ESO-1 immunohistochemistry among mesenchymal myxoid tumors within the differential diagnosis. 

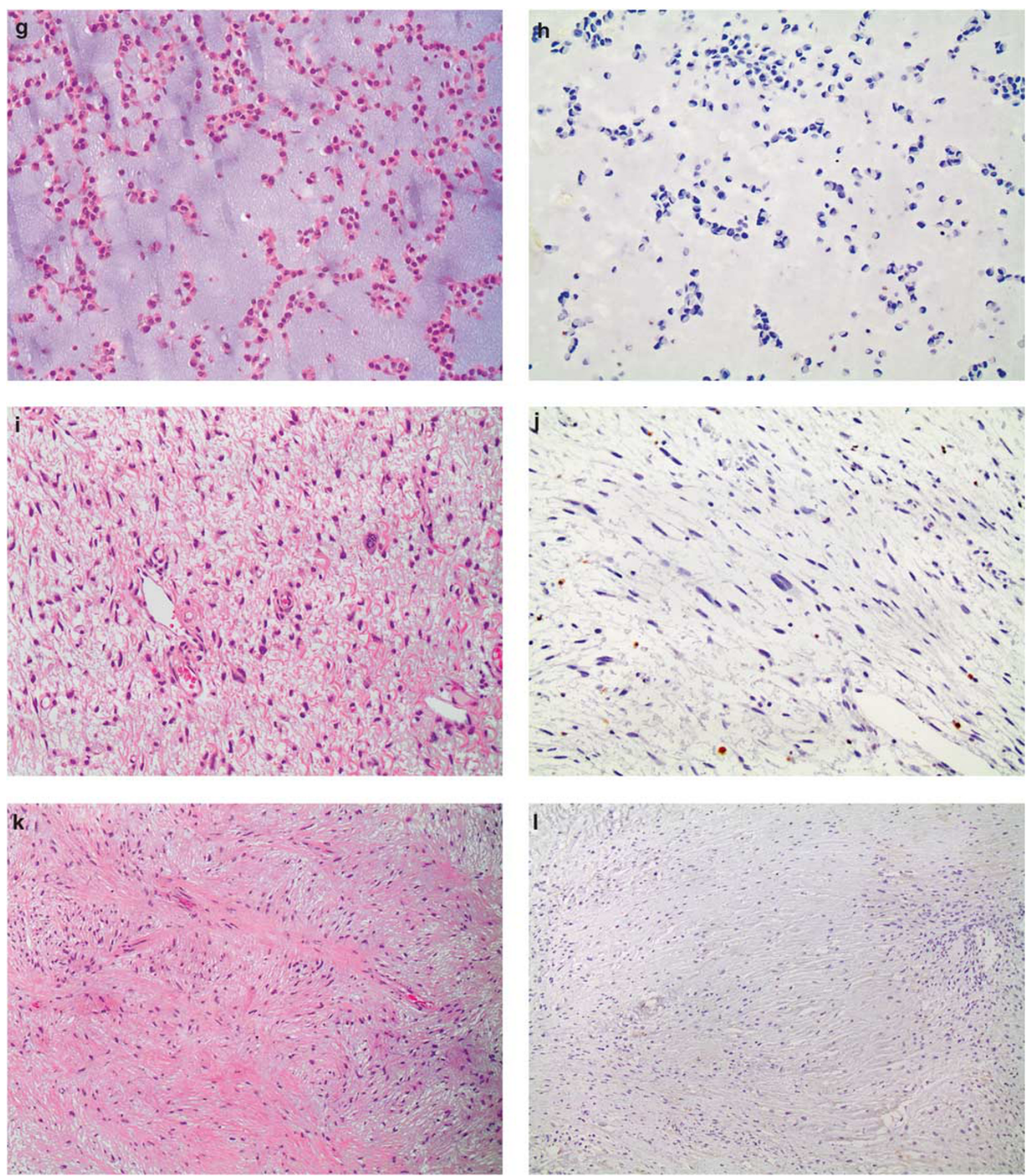

Figure 2 (Continued)

\section{Disclosure/conflict of interest}

The authors declare no conflict of interest.

\section{References}

1 Toro JR, Travis LB, Wu HJ, et al. Incidence patterns of soft tissue sarcomas, regardless of primary site, in the

surveillance, epidemiology and end results program, 1978-2001: an analysis of 26,758 cases. Int J Cancer 2006;119:2922-2930.

2 Weiss SW, JR Goldblum. Liposarcoma, In: Enzinger and Weiss's Soft Tissue Tumors, 5th edn. Mosby/Elsevier Inc: Philadelphia, PA, USA; 2008, pp 477-516.

3 Antonescu CR, Tschernyavsky SJ, Decuseara R, et al. Prognostic impact of P53 status, TLS-CHOP fusion 
transcript structure, and histological grade in myxoid liposarcoma: a molecular and clinicopathologic study of 82 cases. Clin Cancer Res 2001;7:3977-3987.

4 Panagopoulos I, Hoglund M, Mertens F, et al. Fusion of the EWS and CHOP genes in myxoid liposarcoma. Oncogene 1996;12:489-494.

5 Scanlan MJ, Gure AO, Jungbluth AA, et al. Cancer/ testis antigens: an expanding family of targets for cancer immunotherapy. Immunol Rev 2002;188:22-32.

6 Ayyoub M, Taub RN, Keohan ML, et al. The frequent expression of cancer/testis antigens provides opportunities for immunotherapeutic targeting of sarcoma. Cancer Immun 2004;4:7.

7 Chen YT, Chiu R, Lee P, et al. Chromosome X-encoded cancer/testis antigens show distinctive expression patterns in developing gonads and in testicular seminoma. Hum Reprod 2011;26:3232-3243.

8 Nicholaou T, Ebert L, Davis ID, et al. Directions in the immune targeting of cancer: lessons learned from the cancer-testis AgNY-ESO-1. Immunol Cell Biol 2006;84:303-317.

9 Chen YT, Scanlan MJ, Sahin U, et al. A testicular antigen aberrantly expressed in human cancers detected by autologous antibody screening. Proc Natl Acad Sci USA 1997;94:1914-1918.

10 Jungbluth AA, Chen YT, Stockert E, et al. Immunohistochemical analysis of NY-ESO-1 antigen expression in normal and malignant human tissues. Int J Cancer 2001;92:856-860.

11 Pollack SM, Jungbluth AA, Hoch BL, et al. NY-ESO-1 is a ubiquitous immunotherapeutic target antigen for patients with myxoid/round cell liposarcoma. Cancer 2012;118:4564-4570.

12 Hemminger JA, Ewart TA, Scharschmidt TJ, et al. The cancer-testis antigen NY-ESO-1 is highly expressed in myxoid and round cell subset of liposarcomas. Mod Pathol 2013;26:282-288.

13 Chen YT, Boyer AD, Viars CS, et al. Genomic cloning and localization of CTAG, a gene encoding an autoimmunogenic cancer-testis antigen NY-ESO-1, to human chromosome Xq28. Cytogenet Cell Genet 1997;79:237-240.

14 Zendman AJ, Ruiter DJ, Van Muijen GN. Cancer/testisassociated genes: identification, expression profile, and putative function. J Cell Physiol 2003;194: 272-288.

15 Cronwright G, Le Blanc K, Gotherstrom C, et al. Cancer/testis antigen expression in human mesenchymal stem cells: down-regulation of SSX impairs cell migration and matrix metalloproteinase 2 expression. Cancer Res 2005;65:2207-2215.

$16 \mathrm{Xu} \mathrm{H}, \mathrm{Gu} \mathrm{N}$, Liu ZB, et al. NY-ESO-1 expression in hepatocellular carcinoma: a potential new marker for early recurrence after surgery. Oncol Lett 2012;3:39-44.

17 Gure AO, Chua R, Williamson B, et al. Cancer-testis genes are coordinately expressed and are markers of poor outcome in non-small cell lung cancer. Clin Cancer Res 2005;11:8055-8062.

18 Jager E, Gnjatic S, Nagata Y, et al. Induction of primary NY-ESO-1 immunity: CD8 + T lymphocyte and antibody responses in peptide-vaccinated patients with NY-ESO-1 + cancers. Proc Natl Acad Sci USA 2000; 97:12198-12203.

19 Odunsi K, Qian F, Matsuzaki J, et al. Vaccination with an NY-ESO-1 peptide of HLA class I/II specificities induces integrated humoral and $\mathrm{T}$ cell responses in ovarian cancer. Proc Natl Acad Sci USA 2007;104: 12837-12842.

20 Caballero OL, Chen YT. Cancer/testis (CT) antigens: potential targets for immunotherapy. Cancer Sci 2009;100:2014-2021.

21 Robbins PF, Morgan RA, Feldman SA, et al. Tumor regression in patients with metastatic synovial cell sarcoma and melanoma using genetically engineered lymphocytes reactive with NY-ESO-1. J Clin Oncol 2011;29:917-924.

22 Ademuyiwa FO, Bshara W, Attwood K, et al. NY-ESO1 cancer testis antigen demonstrates high immunogenicity in triple negative breast cancer. PloS ONE 2012;7:e38783.

23 Jungbluth AA, Antonescu CR, Busam KJ, et al. Monophasic and biphasic synovial sarcomas abundantly express cancer/testis antigen NY-ESO-1 but not MAGE-A1 or CT7. Int J Cancer 2001;94:252-256.

24 Perez D, Herrmann T, Jungbluth AA, et al. Cancer testis antigen expression in gastrointestinal stromal tumors: new markers for early recurrence. Int J Cancer 2008; 123:1551-1555.

25 Lai JP, Robbins PF, Raffeld M, et al. NY-ESO-1 expression in synovial sarcoma and other mesenchymal tumors: significance for NY-ESO-1-based targeted therapy and differential diagnosis. Mod Pathol 2012; 25:854-858.

26 Woloszynska-Read A, Mhawech-Fauceglia P, Yu J, et al. Intertumor and intratumor NY-ESO-1 expression heterogeneity is associated with promoter-specific and global DNA methylation status in ovarian cancer. Clin Cancer Res 2008;14:3283-3290. 